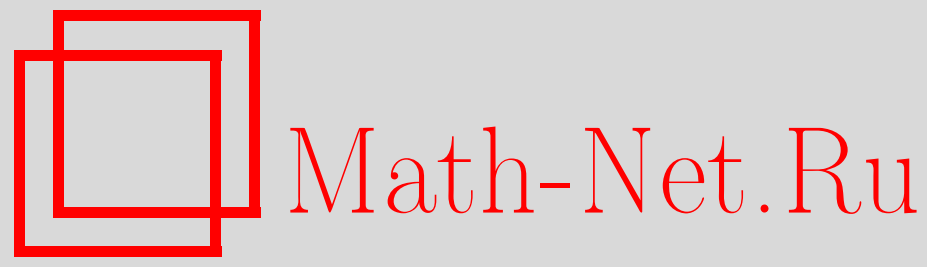

А. А. Глуцюк, В. А. Клепцын, Д. А. Филимонов, И. В. Щуров, О квантовании перемычек в уравнении, моделирующем эффект Джозефсона, Функи. анализ и его прил., 2014, том 48, выпуск 4, 47-64

DOI: https://doi.org/10.4213/faa3161

Использование Общероссийского математического портала MathNet.Ru подразумевает, что вы прочитали и согласны с пользовательским соглашением http://www . mathnet.ru/rus/agreement

Параметры загрузки:

IP : 54.197 .130 .99

26 апреля 2023 г., 14:53:26

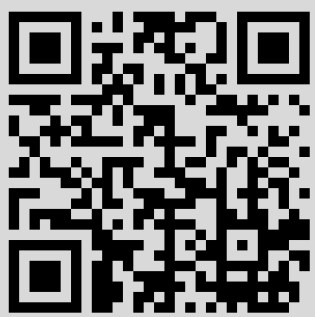




\title{
О квантовании перемычек в уравнении, моделирующем эффект Джозефсона*
}

\author{
(c) 2014. А. А. Глуцюк, В. А. Клепцын, Д. А. Филимонов, И. В. Щуров
}

В статье исследуется двупараметрическое семейство неавтономных обыкновенных дифференциальных уравнений на торе, моделирующее эффект Джозефсона из физики сверхпроводников. Изучается его число вращения как функция от параметров и языки Арнолъда (иначе называемые областями фазового захвата): множества уровня числа вращения, имеющие непустую внутренность. Языки рассматриваемого семейства уравнений обладают рядом нетипичных свойств: они существуют только для целых чисел вращения; границы языков задаются аналитическими кривыми (результаты совместных работ В. М. Бухштабера, О. В. Карпова, С. И. Тертычного и Ю. С. Ильяшенко, Д. А. Рыжова, Д. А. Филимонова). В точках пересечения граничных кривых ширина языка равна нулю: образуются перемычки. Численные эксперименты и теоретические исследования, проделанные в совместных работах В. М. Бухштабера, О. В. Карпова, С. И. Тертычного и А. В. Клименко, О. Л. Ромаскевич, показывают, что каждый язык Арнольда образует бесконечную цепочку примыкающих друг к другу областей, разделенных перемычками и уходящих на бесконечность в асимптотически вертикальном направлении. Недавно в ходе численных экспериментов было также обнаружено, что для каждого языка Арнольда все его перемычки ложатся на одну и ту же вертикальную прямую с целочисленной абсциссой, равной соответствующему числу вращения. В статье приведено доказательство этого факта для некоторого открытого множества рассматриваемых двупараметрических семейств уравнений. В общем случае доказано более слабое утверждение: абсцисса каждой перемычки целочисленна, имеет тот же знак, что и число вращения, и по модулю не превосходит числа вращения. Доказательство основано на представлении рассматриваемых дифференциальных уравнений как проективизаций линейных дифференциальных уравнений на сфере Римана и классической теории линейных уравнений с комплексным временем.

\section{§1. Введение}

1.1. Основные результаты. В статье рассматривается семейство обыкновенных дифференциальных уравнений на торе $\mathbb{T}^{2}=S^{1} \times S^{1}, S^{1}=\mathbb{R} / 2 \pi \mathbb{Z}$, с координатами $(x, t)$, имеющее вид

$$
\dot{x}=\frac{d x}{d t}=\nu \sin x+a+s \sin t, \quad a, \nu, s \in \mathbb{R}, \nu \neq 0 .
$$

* В данной статье использованы результаты, полученные И. В. Щ. в ходе выполнения проекта №11-01-0239 «Инвариантные многообразия и асимптотическое поведение быстромедленных отображений» в рамках программы «Научный фонд НИУ ВШЭ» в 2012/2013/ 2014 гг. Его исследования также частично поддержаны грантом фонда Династия и грантом РФФИ 12-01-31241-мол_а. Исследования А. А. Г. частично поддержаны французскими грантами ANR-08-JCJC-0130-01 и ANR-13-JS01-0010. Исследования всех авторов частично поддержаны совместным грантом РФФИ-CNRS 10-01-93115 НЦНИЛ_а и грантами РФФИ 10-01-00739-a, 13-01-00969-а. 
Это семейство уравнений, которые мы будет для краткости называть уравнениями класса Д, моделирует эффект Джозефсона из физики сверхпроводников. Значение параметра $\nu$ фиксируется произвольным, не равным нулю.

Отображение потока уравнения за период $2 \pi$ есть диффеоморфизм

$$
h_{a, s}: S^{1} \rightarrow S^{1}
$$

пространственной окружности $S^{1}=S^{1} \times\{0\}$. В настоящей статье исследуется его число вращения $\rho=\rho(a, s)$ как функция от параметров $a$ и $s$. (Соглашение о нормировке: число вращения поворота равно углу поворота, деленному на $2 \pi$.) Будем называть координатную ось $a$ горизонтальной (и $a$-координату абсииссой), а ось $s$ вертикальной (и $s$-координату ординатой).

Определение 1.1. Назовем $r$-м языком Арнольда множество уровня $\{(a, s) \mid$ $\rho(a, s)=r\} \subset \mathbb{R}^{2}$, если оно имеет непустую внутренность.

Число вращения системы (1.1) имеет физическую интерпретацию: это среднее напряжение за длительный промежуток времени. Отрезки, по которым языки Арнольда пересекают горизонтальные прямые, соответствуют ступенькам Шапиро на вольт-амперной характеристике. Ранее было показано, что

- языки Арнольда существуют только для целых значений числа вращения $([4],[7],[8])$;

- граница каждого языка $\rho=r$ состоит из двух аналитических кривых, являющихся графиками функций, обозначаемых через $a=g_{r}^{-}(s)$ и $a=g_{r}^{+}(s)$ (см. [3]; А. В. Клименко независимо заметил, что этот факт мгновенно следует из соображений симметрии ${ }^{1)}$ уравнения класса Д, см. [18]);

- каждая функция $g_{r}^{-}(s), g_{r}^{+}(s)$ имеет асимптотику $r$-й функции Бесселя на бесконечности (это численно обнаружено и обосновано на физическом уровне строгости в [16], см. также [10, гл. 5], [13, разд. 11.1], [5]; математически доказано в [18]):

$$
\left\{\begin{array}{l}
g_{r}^{-}(s)=r-\nu J_{r}(-s / \nu)+o\left(s^{-1 / 2}\right), \\
g_{r}^{+}(s)=r+\nu J_{r}(-s / \nu)+o\left(s^{-1 / 2}\right) ;
\end{array}\right.
$$

- таким образом, каждый язык Арнольда есть бесконечная цепочка примыкающих друг к другу ограниченных областей, уходящих на бесконечность в асимптотически вертикальном направлении; точки примыкания соседних областей, не лежащие на оси $s=0$, называются перемычками.

В ходе численных экспериментов был обнаружен следующий

Экспериментальный факт А. Для любого фиксированного $\nu \neq 0$ и любого $r \in \mathbb{Z}$ все перемычки $r$-го языка Арнольда леюат на одной и той же вертикальной прямой $a=r$, см. рис. 1.

Основным результатом настоящей статьи является следующая теорема, которая частично доказывает упомянутый выше экспериментальный факт.

Теорема 1.2. Экспериментальный факт $A$ верен при любом фиксированном $\nu \neq 0$ c $|\nu| \leqslant 1$. При любом фиксированном $\nu \neq 0$ все перемычки имеют целочисленные абсииссы. У каждой перемычки абсиисса имеет тот же знак,

1) Уравнение класса Д имеет симметрию $(x, t) \mapsto(\pi-x, \pi-t)$, в силу которой параболическими неподвижными точками отображения потока за период могут быть только неподвижные точки $\pm \pi / 2$ симметрии, см. [18]. Доказательство эквивалентного утверждения содержится в [15, с. 30]. 


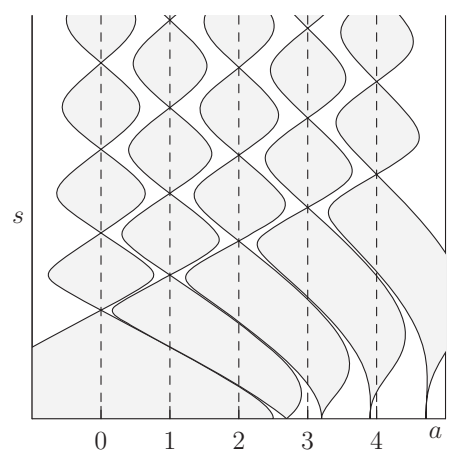

(a) $\nu=2,5$

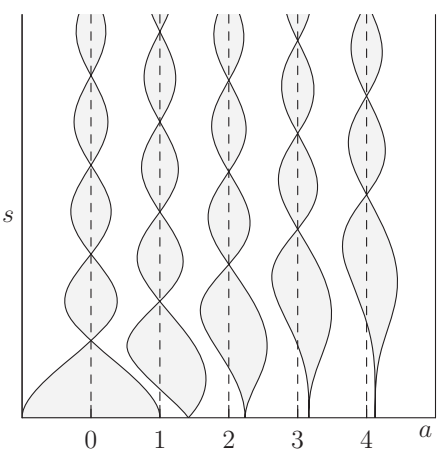

(b) $\nu=1$

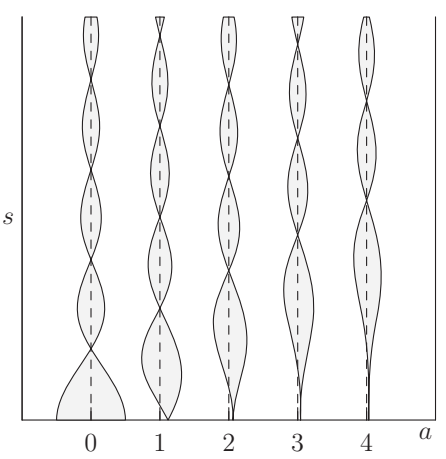

(c) $\nu=0,5$

Рис. 1. Языки Арнольда с номерами 0, 1, 2, 3, 4. Перемычки имеют целочисленные абсциссы, равные номеру языка. Как видно из рисунка, нулевой язык Арнольда пересекает прямую $s=0$ по отрезку ${ }^{1)}[-\nu, \nu]$.

что и соответствующее число вращения, и не превосходит числа вращения по модулю. Перемычки, отвечающие нулевому числу вращения, суть в точности перемычки, лежсащие на оси $a=0$.

Следствие 1.3. Для любых $\nu \neq 0$ u $r \in \mathbb{Z}$ существует $M=M(\nu, r)>0$, такое, что все перемычки $r$-го языка Арнолъда с ординатами, по модулю большими $M$, лежат на прямой $a=r$.

Следствие 1.3 вытекает из целочисленности абсцисс перемычек (теорема 1.2) и асимптотической формулы (1.2) для границы языка Арнольда, в силу которой точки $r$-го языка с достаточно большими по модулю ординатами лежат в 1-окрестности прямой $a=r$.

Замечание 1.4. Известно, что при любом $r \in \mathbb{Z} \backslash 0$ язык Арнольда $\{\rho(a, s)$ $=r\}$ пересекает горизонтальную ось $s=0$ ровно в одной точке с абсциссой $\sqrt{r^{2}+\nu^{2}}$ (см. [8] и [3, следствие 3]). Это точка примыкания соседних компонент внутренности языка, которую мы будем называть ложной перемычкой.

1) Часто вместо семейства уравнений (1.1) рассматривается его перенормировка: двупараметрическое семейство уравнений $\dot{x}+\sin x=B+A \cos (\omega t)$ с фиксированным $\omega \in \mathbb{R}$. В последнем семействе нулевой язык Арнольда пересекает прямую $A=0$ по отрезку $[-1,1]$ при любом $\omega$. 
1.2. Идея доказательства и план статьи. Доказательство теоремы 1.2 представлено в $\S 3$. Оно основано на представлении семейства уравнений класса Д как семейства проективизаций линейных дифференциальных уравнений на сфере Римана (полученном в работах различных авторов, см. также разд. 2.2 ниже) и классической теории линейных уравнений с комплексным временем.

Определение 1.5. Будем называть невырожденный линейный оператор в линейном пространстве проективно тождественным, если он имеет тождественную проективизацию как индуцированное отображение проективного пространства.

Целочисленность абсцисс перемычек при любом $\nu \neq 0$ доказывается в разд. 3.1. Линейные уравнения, соответствующие уравнениям класса Д, имеют две нерезонансные иррегулярные особые точки 0 и $\infty$ ранга Пуанкаре 1 на сфере Римана (см. разд. 2.2). Перемычки отвечают тем значениям параметров, при которых монодромия линейного уравнения проективно тождественна. Оказывается, что это имеет место тогда и только тогда, когда справедливы следующие утверждения:

- росток рассматриваемого линейного уравнения в иррегулярной особой точке 0 приводится аналитической заменой переменных к его формальной нормальной форме (прямой сумме одномерных уравнений);

- монодромия последней проективно тождественна.

Это выводится из классических результатов об аналитической классификации ростков линейных уравнений в нерезонансных иррегулярных особых точках. Матрица вычета нормальной формы имеет единственное ненулевое собственное значение, равное абсциссе перемычки. Отсюда следует целочисленность абсциссы перемычки.

Экспериментальный факт А при $|\nu| \leqslant 1$ доказывается в разд. 3.2. Дополнительное элементарное дифференциальное неравенство (уточнение леммы 4 из [3]) показывает, что при $|\nu| \leqslant 1$ дополнение $r$-го языка Арнольда до горизонтальной оси $s=0$ лежит строго между прямыми $a=r \pm 1$. Таким образом, все его перемычки должны лежать на прямой $a=r$.

Общий случай, когда $\nu$ произвольно, разбирается в разд. 3.3. Легко показать, что абсцисса перемычки имеет тот же знак, что и соответствующее число вращения. Дополнительные рассуждения о соответствующих уравнениях Риккати, использующие принцип аргумента для комплексных решений, доказывают, что модуль абсциссы каждой перемычки не превосходит модуля числа вращения. Это докажет теорему.

Подготовительный материал (число вращения, линейные уравнения, операторы Стокса и монодромия) содержится в 2.

\section{§2. Предварительные сведения}

2.1. Число вращения потока на торе и перемычки. Рассмотрим поток на торе $\mathbb{T}^{2}=S^{1} \times S^{1}=\mathbb{R}^{2} / 2 \pi \mathbb{Z}^{2}$ с координатами $(x, t)$, заданный неавтономным дифференциальным уравнением с гладкой правой частью

$$
\dot{x}=\frac{d x}{d t}=f(x, t) .
$$


Преобразование потока за время $t$ есть диффеоморфизм пространственной окружности, который мы обозначим через $h_{t}: S^{1} \rightarrow S^{1}$. Рассмотрим универсальную накрывающую над пространственной окружностью:

$$
\mathbb{R} \rightarrow S^{1}=\mathbb{R} / 2 \pi \mathbb{Z}
$$

Преобразования потока уравнения (2.1) поднимаются на универсальную накрывающую и индуцируют диффеоморфизмы

$$
H_{q, t}: \mathbb{R}=\mathbb{R} \times\{q\} \rightarrow \mathbb{R}=\mathbb{R} \times\{q+t\}, \quad H_{q, 0}=\mathrm{Id} .
$$

Напомним, что для любых $(x, q) \in \mathbb{R} \times S^{1}$ предел

$$
\rho=\lim _{n \rightarrow+\infty} \frac{1}{n} H_{q, 2 \pi n}(x) \in \mathbb{R}
$$

существует, не зависит ни от $q$, ни от $x$ и называется числом вращения потока уравнения (2.1) (см., например, [1, с. 124]).

Рассмотрим теперь (произвольное) аналитическое семейство уравнений

$$
v_{a, s}: \quad \dot{x}=g(x, t, s)+a, \quad a, s \in \mathbb{R} .
$$

Предложение 2.1. Число вращения $\rho=\rho(a, s)$ потока (2.3) есть непрерьвная функиия от параметров $(a, s)$, которая не убывает как функиия от а. Ecли при некотором значении параметров $\left(a_{0}, s_{0}\right)$ преобразование потока $h_{2 \pi}=$ $h_{a_{0}, s_{0}, 2 \pi}: S^{1} \times\{0\} \rightarrow S^{1} \times\{0\}$ уравнения (2.3) имеет неподвижную точку, то число вращения $\rho\left(a_{0}, s_{0}\right)$ иелочисленно. Если в последнем случае, дополнительно, преобразование потока $h_{2 \pi}$ не является тождественным, то существует такой интервал I с кониом $a_{0}$, что

- для любого $a \in I$ преобразование $h_{a, s_{0}, 2 \pi}$ имеет хотя бы одну неподвиюную точку;

- для любого $a \in I$ потоки всех векторных полей, достаточно $C^{1}$-близких $\kappa v_{a, s_{0}}$, имеют то же число вращения $\rho\left(a_{0}, s_{0}\right)$.

Доказательство. Первое и второе утверждения предложения содержатся в [1, с. 130-133]. Докажем третье утверждение. Пусть преобразование потока $h=h_{a_{0}, s_{0}, 2 \pi}$ не является тождественным и имеет неподвижную точку $O \in S^{1}$. Тогда последняя - изолированная неподвижная точка в силу аналитичности. Пусть $J=[P, O] \subset S^{1}$ - отрезок с концом $O$ слева от $O$, не содержащий других неподвижных точек. Тогда $h(J) \neq J$ : либо $h(J) \subset J$, либо $h(J) \supset J$. Без ограничения общности мы рассмотрим только первый случай: $h_{a, s_{0}, 2 \pi}(J) \subset J$ при $a=a_{0}$. Существует интервал $I=\left(c, a_{0}\right) \subset \mathbb{R}$, такой, что последнее включение выполнено для любого $a \in I$ и является строгим, $h_{a, s_{0}, 2 \pi}(J) \subset \operatorname{Int}(J)$, так как для любых фиксированных $s, t$ и $x$ функция $a \mapsto h_{a, s, t}(x)$ непрерывна и строго возрастает. Тем самым, для любого $a \in I$ отображение $h_{a, s_{0}, 2 \pi}$ переводит отрезок $J$ внутрь себя, а значит, имеет там неподвижную точку и, следовательно, целое число вращения, равное $\rho\left(a_{0}, s_{0}\right)$. Последнее включение выживает при любых $C^{0}$-малых возмущениях отображения $h_{a, s_{0}, 2 \pi}$. Это вместе с непрерывностью числа вращения доказывает предложение 2.1.

Рассмотрим теперь семейство (1.1) уравнений класса Д. Для каждых значений параметров $(a, s)$ обозначим через

$$
h_{a, s}=h_{a, s, 2 \pi}: S^{1} \times\{0\} \rightarrow S^{1} \times\{0\}
$$


преобразование потока соответствующего уравнения за период $2 \pi$. Пусть

$$
\rho(a, s) \text { обозначает соответствующее число вращения. }
$$

Предложение 2.2. Число вращения $\rho(a, s)$ есть непрерьвная функиия от параметров $(a, s)$, которая не убъвает как функиия от а. Перемъчки языков Арнольда и ложные перемычки (см. введение) отвечают в точности тем значениям параметров, при которых соответствующее преобразование потока $h_{a, s}$ тождественно.

Доказательство. Первое утверждение предложения 2.2 следует из первого утверждения предложения 2.1. Докажем второе утверждение. Предположим противное: для одной из перемычек $\left(a_{0}, s_{0}\right)$ (настоящей или ложной) соответствующее преобразование потока не является тождественным. Оно имеет целочисленное число вращения и, тем самым, неподвижную точку. Следовательно, имеется интервал $I \subset \mathbb{R}$, примыкающий к $a_{0}$, такой, что для любого $a \in I$ преобразование $h_{a, s_{0}}$ имеет неподвижную точку и интервал $I \times\left\{s_{0}\right\} \subset\left\{s=s_{0}\right\}$ лежит во внутренности языка Арнольда $\left\{\rho(a, s)=\rho\left(a_{0}, s_{0}\right)\right\}$ (предложение 2.1). В то же время прямая $s=s_{0}$ не может пересекать внутренности языка. Действительно, в противном случае близкая ей параллельная прямая пересекла бы, как минимум, две различные компоненты языка (по определению перемычки), что противоречило бы монотонности числа вращения как функции от $a$. Полученное противоречие доказывает тождественность преобразования потока. И обратно, пусть преобразование потока тождественно. Тогда рассматриваемая точка $\left(a_{0}, s_{0}\right)$ принадлежит целочисленному языку Арнольда. Она не может лежать в его внутренности. Действительно, при увеличении (уменьшении) параметра $a$ при фиксированном $s=s_{0}$ образы всех точек $x \in S^{1}$ под действием преобразования потока движутся в положительную (отрицательную) сторону. В частности, у возмущенного отображения потока не будет неподвижных точек (в силу тождественности невозмущенного). Таким образом, точка $\left(a_{0}, s_{0}\right)$ горизонтальной прямой $\mathbb{R} \times\left\{s_{0}\right\}$ является изолированной точкой пересечения этой прямой с языком Арнольда $\left\{\rho(a, s)=\rho\left(a_{0}, s_{0}\right)\right\}$. Следовательно, она должна быть перемычкой (настоящей или ложной). Предложение доказано.

2.2. Сведение уравнений класса Д к уравнениям Риккати. Результаты этого раздела в несколько иных терминах ранее были получены в работах [6], [8], [14].

Предложение 2.3. Замена переменных

$$
p=e^{i x}, \quad \tau=e^{i t}
$$

сводит семейство уравнений класса Д к семейству уравнений вида Риккати

$$
\frac{d p}{d \tau}=\frac{1}{\tau^{2}}\left(\nu\left(1-p^{2}\right) \frac{i \tau}{2}+\left(a \tau+\frac{i s\left(1-\tau^{2}\right)}{2}\right) p\right) .
$$

Последнее получается из следующего семейства линейных обыкновенных дифференциальных уравнений проективизацией:

$$
\dot{z}=\frac{A(\tau)}{\tau^{2}} z, \quad z=\left(z_{1}, z_{2}\right) \in \mathbb{C}^{2}, A(\tau)=\left(\begin{array}{cc}
0 & \frac{i \nu \tau}{2} \\
\frac{i \nu \tau}{2} & \frac{i s}{2}\left(1-\tau^{2}\right)+a \tau
\end{array}\right), \quad p=\frac{z_{2}}{z_{1}} .
$$


Доказательство. Подставляя замену (2.6) в уравнение (1.1), получаем

$$
\begin{gathered}
\sin x=\frac{1}{2 i}\left(p-p^{-1}\right), \quad \sin t=\frac{1}{2 i}\left(\tau-\tau^{-1}\right), \\
\dot{p}=\frac{d p}{d t}=i p \dot{x}=\frac{\nu p}{2}\left(p-p^{-1}\right)+i a p+\frac{s}{2}\left(\tau-\tau^{-1}\right) p, \quad \dot{\tau}=\frac{d \tau}{d t}=i \tau .
\end{gathered}
$$

Следовательно,

$$
\frac{d p}{d \tau}=\frac{\dot{p}}{\dot{\tau}}=\frac{1}{\tau^{2}}\left(\frac{i \nu \tau}{2}\left(1-p^{2}\right)+a p \tau+\frac{i s}{2}\left(1-\tau^{2}\right) p\right),
$$

и, тем самым, мы получаем уравнение (2.7). Последнее есть уравнение вида Риккати и, значит, является проективизацией линейного уравнения

$$
\dot{z}=\frac{d z}{d \tau}=\frac{B(\tau)}{\tau^{2}} z, \quad z=\left(z_{1}, z_{2}\right) \in \mathbb{C}^{2}, p=\frac{z_{2}}{z_{1}} .
$$

Уравнение (2.9) однозначно определено с точностью до нормировки, так как соответствующая вектор-функция $z(\tau)$ определяется решением $p(\tau)$ уравнения Риккати однозначно с точностью до умножения на скалярную функцию от $\tau$. Найдем коэффициенты уравнения (2.9). Подставляя его в формулу $p=z_{2} / z_{1}$ и дифференцируя последнюю, получим

$$
\frac{d p}{d \tau}=\frac{1}{\tau^{2}}\left(\left(B_{22}(\tau)-B_{11}(\tau)\right) p+B_{21}(\tau)-B_{12}(\tau) p^{2}\right)
$$

Сравнение последнего уравнения с уравнением (2.7) дает

$$
B_{22}(\tau)-B_{11}(\tau)=a \tau+\frac{i s}{2}\left(1-\tau^{2}\right), \quad B_{21}(\tau)=B_{12}(\tau)=\frac{i \nu \tau}{2} .
$$

Всякая матричнозначная функция $B=\left(B_{i j}\right)(\tau)$, удовлетворяющая предыдущим равенствам, определяет линейное уравнение, отвечающее уравнению Риккати (2.7). Функция $B_{11}(\tau)$ может быть выбрана произвольной, и ее выбор определяет матрицу $B(\tau)$ однозначно. Положив $B_{11} \equiv 0$, получим матричнозначную функцию $A(\tau)$ из (2.8). Предложение доказано.

2.3. Иррегулярные особые точки линейных дифференциальных уравнений: явление Стокса и монодромия. Все результаты, представленные в настоящем разделе, являются классическими и содержатся в [2], [9], [12], [17], [19].

Рассмотрим росток линейного обыкновенного дифференциального уравнения

$$
\dot{z}=\frac{B(\tau)}{\tau^{2}} z, \quad z=\left(z_{1}, z_{2}\right) \in \mathbb{C}^{2},
$$

в окрестности нерезонансной иррегулярной особой точки $\tau=0$ ранга 1 . По определению это в точности означает, что $B(\tau)$ есть голоморфная в нуле $(2 \times 2)$ матричнозначная функция и матрица $B(0)$ имеет различные собственные значения $\lambda_{1}, \lambda_{2}$. Без ограничения общности будем считать матрицу $B(0)$ диагональной,

$$
B(0)=\operatorname{diag}\left(\lambda_{1}, \lambda_{2}\right), \quad \lambda_{2}-\lambda_{1} \in i \mathbb{R}_{+} .
$$

Этого можно добиться, применив линейные постоянные замены переменных $z$ и $\tau$. 
Определение 2.4. Два ростка уравнений вида (2.10) аналитически (формально) эквивалентны, если существует замена $z=H(\tau) w$ переменной $z$, где $H(\tau)$ - голоморфная обратимая матричнозначная функция (соответственно формальный обратимый степенной ряд по $\tau$ с матричными коэффициентами), преобразующая одно уравнение в другое.

Аналитическая классификация иррегулярных нерезонансных ростков линейных уравнений (2.10) и результаты, представленные в настоящем разделе, были получены в классических работах [12], [17], [19], а также представлены в [2] и [9]. Оказывается, что всякий росток уравнения (2.10) формально эквивалентен единственной прямой сумме одномерных уравнений вида

$$
\left\{\begin{array}{l}
\dot{w}_{1}=\frac{b_{10}+b_{11} \tau}{\tau^{2}} w_{1}, \\
\dot{w}_{2}=\frac{b_{20}+b_{21} \tau}{\tau^{2}} w_{2},
\end{array} \quad b_{20}-b_{10} \in i \mathbb{R}_{+},\right.
$$

называемой формальной нормальной формой; здесь $b_{j 0}=\lambda_{j}$. Соответствующий нормализующий ряд $H(\tau), z=H(\tau) w$, единствен с точностью до умножения справа на постоянную диагональную матрицу. Как правило, нормализующий ряд расходится. В то же время существует покрытие проколотой окрестности нуля двумя секторами $S_{0}$ и $S_{1}$ с вершиной в нуле на комплексной прямой $\tau$, обладающее следующим свойством. Над каждым сектором $S_{j}$ существует единственная замена переменных $z=H_{j}(\tau) w$, которая преобразует $(2.10)$ в $(2.11)$, где $H_{j}(\tau)$ - аналитическая обратимая матричнозначная функция на $S_{j}$, $C^{\infty}$-гладко продолжающаяся на замыкание $\bar{S}_{j}$ сектора, так что ее асимптотический ряд Тейлора в 0 существует и совпадает с данным нормализующим рядом. Предыдущее утверждение о существовании и единственности секториальной нормализации справедливо в любом хорошем секторе (см. следующее определение); покрытие состоит из хороших секторов.

Определение 2.5. Назовем сектор в $\mathbb{C}$ с вершиной в 0 хорошим, если он содержит только одну из вещественных полуосей $\mathbb{R}_{ \pm}$и его замыкание не содержит другую (см. рис. 2).

Будем считать, что сектор $S_{0}$ содержит вещественную положительную полуось, а сектор $S_{1}$ - отрицательную, см. рис. 2. Положим $S_{2}=S_{0}$.

Стандартное разложение нормальной формы (2.11) в прямую сумму одномерных уравнений определяет канонический базис в пространстве ее решений (единственным образом, с точностью до умножения базисных функций на константы) с диагональной фундаментальной матрицей. Обозначим эту фундаментальную матрицу через

$$
W(\tau)=\operatorname{diag}\left(w_{1}, w_{2}\right) .
$$

Вместе с нормализующими заменами $H_{j}$ в $S_{j}$ она определяет канонические базисы $\left(f_{j 1}, f_{j 2}\right)$ в пространствах решений уравнения $(2.10)$ в секторах $S_{j}$ с фундаментальными матрицами

$$
Z^{j}(\tau)=H_{j}(\tau) W(\tau), \quad j=0,1,2,
$$

где для любого $j=0,1$ ветвь с индексом $j+1$ фундаментальной матрицы $W(\tau)$ на $S_{j+1}$ получается из ее ветви с индексом $j$ на $S_{j}$ с помощью аналитического продолжения против часовой стрелки. Ветвь фундаментальной матрицы $W$ с 
индексом 2 на $S_{2}=S_{0}$ получается из ветви с индексом 0 умножением справа на матрицу монодромии формальной нормальной формы (2.11). В связной компоненте пересечения $S_{j} \cap S_{j+1}$ имеются два канонических базиса решений, приходящих из секторов $S_{j}$ и $S_{j+1}$. Как правило, они не совпадают. Переход между ними определяется при помощи постоянной матрицы $C_{j}$ :

$$
Z^{j+1}(\tau)=Z^{j}(\tau) C_{j}
$$

Операторы переходов (матрицы $C_{j}$ ) называются операторами (матрииами) Cmокса (см. упомянутую выше литературу). Нетривиальность операторов Стокса доставляет препятствие к аналитической эквивалентности уравнения (2.10) его формальной нормальной форме (2.11) и называется явлением Стокса.

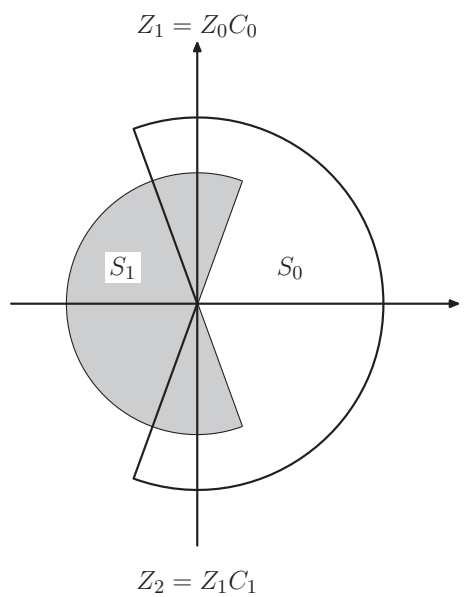

Рис. 2. Секторы $S_{0}, S_{1}$ и операторы Стокса $C_{0}$ и $C_{1}$ над их пересечениями

Замечание 2.6. Матрицы Стокса (2.13) однозначно определены с точностью до одновременного сопряжения с помощью одной и той же диагональной матрицы. Матрицы Стокса унипотентны. Матрица $C_{0}$, отвечающая верхней компоненте пересечения секторов, нижнетреугольная, а матрица $C_{1}$ верхнетреугольная:

$$
C_{0}=\left(\begin{array}{cc}
1 & 0 \\
c_{0} & 1
\end{array}\right), \quad C_{1}=\left(\begin{array}{cc}
1 & c_{1} \\
0 & 1
\end{array}\right) .
$$

Теорема 2.7 ([2], [9], [12], [17], [19]). Уравнение (2.10) аналитически эквивалентно своей формальной нормальной форме (2.11), если и только если оно имеет тривиальные операторь Стокса. Два уравнения (2.10) аналитически эквивалентны, если и только если они имеют одинаковые формалъные нормальные формы и наборы матрии, Стокса (с точностью до упомянутого выше одновременного сопряжения с помощъю диагональной матрищъ).

Пусть $\gamma:[0,1] \rightarrow \mathbb{C} \backslash 0$ - замкнутый путь на проколотой прямой $\tau$, делающий один обход вокруг нуля против часовой стрелки. Напомним, что оператор монодромии уравнения (2.10) - это линейный оператор, действующий в пространстве локальных решений в окрестности точки $\gamma(0)$ и переводящий каждое решение в результат его аналитического продолжения вдоль пути $\gamma$. 
Лемма 2.8 [9, с. 35]. Матрицы монодромии уравнения (2.10) и его формальной нормальной формы (2.11), обозначаемые соответственно через $M u M_{N}$, и введенные выше матрицы Стокса $C_{0}$ и $C_{1}$ удовлетворяют равенству

$$
M=M_{N} C_{1}^{-1} C_{0}^{-1} .
$$

Рассмотрим проективизацию уравнения (2.10) - соответствующее уравнение Риккати на $\overline{\mathbb{C}}$-значную функцию $p(\tau)=z_{2}(\tau) / z_{1}(\tau)$, получаемое из $(2.10)$ с помощью тавтологической проекции $\mathbb{C}^{2} \backslash 0 \rightarrow \overline{\mathbb{C}}=\mathbb{C P}^{1}$. В конце статьи мы будем использовать следующие свойства проективизированных канонических базисных решений уравнения (2.10).

Предложение 2.9. Пусть уравнение (2.10) аналитически эквивалентно своей формальной нормальной форме. Тогда проективизации его канонических базисных решений суть единственные решения $p(\tau)$ соответствующего уравнения Риккати, голоморфные в окрестности нуля. Их значения в нуле равны соответственно нулю и бесконечности в проективной координате $p=z_{2} / z_{1}$.

Предложение 2.9 хорошо известно специалистам и следует из своей справедливости для формальной нормальной формы.

Предложение 2.10. В условиях предложения 2.9 пусть $\psi_{1}(\tau), \psi_{2}(\tau)-$ рассматриваемые канонические решения уравнения Риккати, $\psi_{1}(0)=0, \psi_{2}(0)=$ $\infty, a$

$$
z=H(\tau) w, \quad H(\tau)=\left(\begin{array}{ll}
h_{11} & h_{12} \\
h_{21} & h_{22}
\end{array}\right)(\tau)
$$

- нормализующая замена переменных, приводящая линейное уравнение (2.10) к нормальной форме. Тогда

$$
\psi_{j}=\frac{h_{2 j}}{h_{1 j}}, \quad j=1,2 .
$$

Предложение 2.10 следует из диагональности канонической фундаментальной матрицы решений нормальной формы.

\section{§3. Число вращения и монодромия: доказательство теоремы 1.2}

3.1. Целочисленность перемычек. Рассмотрим уравнения (1.1) класса Д и соответствующие им уравнения Риккати (2.7) и линейные уравнения (2.8). Преобразования потока $h_{a, s}: S^{1} \rightarrow S^{1}$ продолжаются как мёбиусовы преобразования сферы Римана $\overline{\mathbb{C}}=\mathbb{C P}^{1}$ и совпадают с преобразованием монодромии соответствующих уравнений Риккати при обходе вокруг точки $0 \in \overline{\mathbb{C}}$ в силу предложения 2.3. Обозначим через $M_{a, s}$ оператор монодромии линейного уравнения (2.8). По определению справедливо следующее

Предложение 3.1. Продолженные преобразования потока $h_{a, s}$ совпадают c проективизациями операторов монодромии $M_{a, s}$ соответствующих линейных уравнений (2.8).

Росток каждого из линейных уравнений (2.8) в точке 0 иррегулярен и нерезонансен, точнее, имеет вид (2.10). Его формальная нормальная форма имеет вид

$$
\left\{\begin{array}{l}
\dot{w}_{1}=0, \\
\dot{w}_{2}=\tau^{-2}(i s / 2+a \tau) w_{2} .
\end{array}\right.
$$


Оператор монодромии соответствующей формальной нормальной формы будем обозначать через $M_{N, a, s}$. Имеем

$$
M_{N, a, s}=\operatorname{diag}\left(1, e^{2 \pi i a}\right) .
$$

Предложение 3.2. Точка $(a, s)$ отвечает перемычке, если и только если $s \neq 0$ и соответствующий оператор монодромии $M_{a, s}$ проективно тождествен, т.е. является произведением тождественного оператора на скалярный множстель.

Доказательство. Точка отвечает перемычке, если и только если $s \neq 0$ и соответствующее преобразование потока уравнения класса Д за период тождественно в силу предложения 2.2. Это вместе с предложением 3.1 доказывает предложение 3.2 .

Лемма 3.3. Точка $(a, s)$ отвечает перемычке, если и только если $а \in \mathbb{Z}$, $s \neq 0$ и росток в нуле соответствующего линейного уравнения (2.8) аналитически эквивалентен его формальной нормальной борме (3.1).

Доказательство. Пусть $s \neq 0, a \in \mathbb{Z}$ и уравнение (2.8) аналитически эквивалентно своей нормальной форме. Тогда точка $(a, s)$ отвечает перемычке в силу предложения 3.2 и тождественности монодромии (3.2). Докажем, что верно и обратное. Пусть точка $(a, s), s \neq 0$, отвечает перемычке. Тогда оператор $M_{a, s}$ проективно тождествен, а значит, его матрица в любом базисе должна иметь тривиальные наддиагональные и поддиагональные элементы. С другой стороны, его матрица в каноническом базисе пространства решений в сектоpe $S_{0}$, см. рис. 2 , есть произведение диагональной матрицы монодромии $(3.2)$ формальной нормальной формы и двух матриц, обратных к матрицам Стокса, см. (2.15). Последние две матрицы унипотентны, одна из них нижнетреугольная, а другая верхнетреугольная. Произведение трех указанных выше матриц имеет тривиальные недиагональные элементы, если и только если две последние треугольные матрицы имеют тривиальные недиагональные элементы, и, таким образом, тождественны в силу унипотентности. Это в точности означает, что матрицы Стокса тождественны, и, таким образом, линейное уравнение (2.8) аналитически эквивалентно своей формальной нормальной форме по теореме 2.7. В частности, монодромия уравнения (2.8) задается матрицей (3.2), которая проективно тождественна, если и только если $a \in \mathbb{Z}$. Итак, мы показали, что оператор монодромии уравнения (2.8) проективно тождествен, если и только если уравнение аналитически эквивалентно своей формальной нормальной форме и $a \in \mathbb{Z}$. Это вместе с предложением 3.2 доказывает лемму 3.3. Целочисленность абсцисс перемычек доказана.

3.2. Случай $|\nu| \leqslant 1$ : перемычки $r$-го языка лежат на прямой $a=r$. Итак, мы показали, что все перемычки лежат на вертикальных прямых с целочисленными абсциссами. Теперь покажем, что при $|\nu| \leqslant 1$ перемычки каждого индивидуального языка Арнольда лежат на одной и той же целочисленной вертикальной прямой, точнее,

$$
\rho(a, s)=a \text { для каждой перемычки }(a, s) \text {. }
$$

Итак, ниже считаем, что $|\nu| \leqslant 1$. 
Предложение 3.4. При $|\nu| \leqslant 1$ для любых $a, s \in \mathbb{R}$ имеют место неравенства $a-1 \leqslant \rho(a, s) \leqslant a+1$. Оба неравенства являются строгими, за исключением случая, когда $s=0, a= \pm 1$.

Неравенства из предложения 3.4 содержатся в статье [3] и сформулированы там как лемма 4. Приведенное ниже доказательство строгости неравенств повторяет доказательство из статьи [3] с небольшим дополнением.

Доказательство предложения 3.4. Всякое решение $x(t)$ уравнения класса Д удовлетворяет дифференциальным неравенствам

$$
a+s \sin t-1 \leqslant \dot{x}=\nu \sin x+a+s \sin t \leqslant a+s \sin t+1 .
$$

В случае, когда $\sin x \not \equiv \pm 1$, эти неравенства являются строгими при почти всех $t$, а именно при всех $t$, для которых $\sin x(t) \neq \pm 1$. Значит, приращение решения $x(t)$ на любом отрезке $I$ длины $2 \pi$ находится между интегралами по тому же отрезку от крайних частей из строки неравенств (3.4) (см. теорему Чаплыгина о сравнении [11]). Последние интегралы равны соответственно $a-1$ и $a+1$. Отсюда следуют неравенства из предложения. Пусть теперь $\sin x(t) \not \equiv 1$. Тогда неравенства (3.4) являются строгими при почти всех $t$. Отсюда вытекает, что предыдущее приращение находится строго между числами $a \pm 1$ и равномерно отделено от них по всем отрезкам $I$ длины $2 \pi$. Таким образом, число вращения также лежит строго между числами $a \pm 1$ по определению и в силу равномерности. Пусть теперь одно из неравенств (3.4) не является строгим. Тогда в силу предыдущего $\sin x(t) \equiv \pm 1$, а значит, $x(t) \equiv \pi / 2+\pi k, k \in \mathbb{Z}$. Уравнение (1.1) класса Д имеет постоянное решение $x(t)$ с $\sin x(t) \equiv \pm 1$, если и только если $s=0, a=\mp 1$; в этом случае число вращения равно нулю. Предложение доказано.

Рассмотрим язык Арнольда, отвечающий данному целому числу вращения $r$. Его дополнение до горизонтальной оси $s=0$ лежит строго между прямыми $a=r \pm 1$ в силу предыдущего предложения. Его перемычки не лежат на горизонтальной оси (по определению) и имеют целочисленные абсциссы, как показано выше. Значит, они лежат на прямой $a=r$. Первое утверждение теоремы 1.2 доказано.

3.3. Случай произвольного $\nu$ : модуль абсциссы перемычки не превосходит модуля числа вращения. Здесь доказывается второе утверждение теоремы 1.2: при любом фиксированном $\nu \neq 0$ абсцисса каждой перемычки имеет тот же знак, что и соответствующее число вращения, и ее модуль не превосходит модуля числа вращения.

Сначала мы докажем совпадение знаков, которое легко следует из того, что вся ось $s$ лежит в нулевом языке Арнольда (следующее предложение). Затем мы докажем сформулированное выше неравенство, используя комплексные уравнения Риккати и принцип аргумента для их канонических решений.

Предложение 3.5. При $a=0$ и любых $\nu$ и s уравнение (1.1) имеет нулевое число вращения. Таким образом, при любом $\nu \neq 0$ вся осъ $s$ лежит в нулевом языке Арнольда.

Доказательство. При $a=0$ уравнение (1.1) имеет симметрию $(x, t) \mapsto$ $(-x, t+\pi)$.

Значит, если $x(t)$ - решение, то и $\widetilde{x}(t)=-x(t+\pi)$ - тоже решение. Отсюда следует, что инволюция $x \mapsto-x$ сопрягает отображения потока $H_{0,2 \pi}: \mathbb{R} \times\{0\} \rightarrow$ 
$\mathbb{R} \times\{0\}$ и $H_{\pi, 2 \pi}: \mathbb{R} \times\{\pi\} \rightarrow \mathbb{R} \times\{\pi\}$ поднятого уравнения (1.1). Следовательно, рассматриваемые отображения имеют противоположные числа вращения, определенные как пределы (2.2). С другой стороны, их числа вращения равны числу вращения потока, см. разд. 2.1. Итак, рассматриваемые числа вращения одновременно равны и противоположны. Следовательно, число вращения равно нулю. Предложение доказано.

Следствие 3.6. Абсиисса каждой перемъчки имеет тот же знак, что и соответствующее число вращения, если последнее не равно нулю.

Доказательство. Число вращения равно нулю при $a=0$ и не убывает как функция от $a$. Это доказывает следствие.

Фиксируем $\nu \neq 0$ и рассмотрим произвольную перемычку $(a, s)$. Пусть $\rho=$ $\rho(a, s)$ - соответствующее число вращения. Напомним, что соответствующее линейное уравнение (2.8) аналитически эквивалентно своей формальной нормальной форме в нуле (лемма 3.3 ). Отсюда следует, что $\overline{\mathbb{C}}$-значные проективизированные канонические базисные решения уравнения (2.8) голоморфны в полной окрестности нуля (предложение 2.9), а следовательно, на всей прямой $\mathbb{C}$. Обозначим последние проективизированные решения через $\psi_{1}(\tau), \psi_{2}(\tau)$, так что $\psi_{1}(0)=0, \psi_{2}(0)=\infty$, см. предложение 2.9. Положим

$$
S_{p}^{1}=\{|p|=1\} \subset \overline{\mathbb{C}}, S_{\tau}^{1}=\{|\tau|=1\} \subset \overline{\mathbb{C}}, \mathbb{T}^{2}=S_{p}^{1} \times S_{\tau}^{1}, D_{1}=\{|\tau|<1\} .
$$

Замечание 3.7. Тор $\mathbb{T}^{2}$ инвариантен относительно уравнения Риккати (2.7), ограниченного на $\overline{\mathbb{C}} \times S_{\tau}^{1}$. Функции $\psi_{1}, \psi_{2}$ суть его различные решения, а значит, $\psi_{1}(\tau) \neq \psi_{2}(\tau)$ при всех $\tau \in \mathbb{C}$. Ограничение каждой из них на единичную окружность $S_{\tau}^{1}$ принимает значения, лежащие либо все по одну сторону от единичной окружности $S_{p}^{1}$, либо на самой окружности. Это следует из инвариантности тора $\mathbb{T}^{2}$.

Доказательство неравенства $|a| \leqslant|\rho|$ для перемычек из теоремы 1.2 основано на следующей формуле для числа вращения $\rho$.

Лемма 3.8. Пусть $\left|\psi_{1}\right|_{S_{\tau}^{1}} \leqslant 1$. Тогда

$$
\rho=a-2 \#\left(\text { полюсъь функиии }\left.\psi_{1}\right|_{D_{1}}\right) \text {. }
$$

Доказательство. Для произвольного решения $\psi$ уравнения Риккати (2.7) и любого $r>0$, такого, что $\left.\psi\right|_{\{|\tau|=r\}} \neq \infty$, рассмотрим уравнение в вариациях вдоль решения $\psi$. Заметим, что решение уравнения в вариациях однозначно определено с точностью до мультипликативной константы и голоморфно по $\tau$ в окрестности окружности $\{|\tau|=r\}$. Это следует из того, что уравнение Риккати имеет тривиальную монодромию, как и соответствующее линейное уравнение (предложение 3.2). Фиксируем произвольное ненулевое решение $v(\tau)$ уравнения в вариациях. Ориентируем окружность $\{|\tau|=r\}$ против часовой стрелки и введем индекс

$\chi_{r}(\psi)=$ приращение аргумента решения $v(\tau)$ вдоль окружности $\{|\tau|=r\}$.

Предложение 3.9. Для любого достаточно малого $r>0$ имеем $\chi_{r}\left(\psi_{1}\right)=a$.

Справедливость предложения следует из его справедливости для уравнения Риккати, являющегося проективизацией формальной нормальной формы (3.1), и аналитической нормализуемости рассматриваемого линейного уравнения (2.8). 
Предложение 3.10. Пусть $\left.\left|\psi_{1}\right|\right|_{S_{\tau}^{1}} \leqslant 1$. Тогда $\chi_{1}\left(\psi_{1}\right)=\rho$.

Доказательство. Фиксируем $\tau_{0} \in S_{\tau}^{1}$. Преобразования потока $\overline{\mathbb{C}} \times\left\{\tau_{0}\right\} \rightarrow$ $\overline{\mathbb{C}} \times\{\tau\}$ уравнения Риккати вдоль единичной окружности $|\tau|=1$ суть мёбиусовы преобразования, сохраняющие единичный диск. Индекс $\chi_{1}(\psi)$ любого решения уравнения Риккати с $\psi\left(\tau_{0}\right) \in \overline{D_{1}}$ не зависит от выбора решения и равен $\rho$ при $\psi\left(\tau_{0}\right) \in S_{p}^{1}$. Это доказывает предложение.

Предложение 3.11. Рассмотрим произвольное уравнение Риккати. Пусть $0<r_{1}<r_{2}$ таковы, что уравнение Риккати голоморфно в окрестности замкнутого кольиа $A_{r_{1} r_{2}}=\left\{r_{1} \leqslant|\tau| \leqslant r_{2}\right\}$ и имеет там мероморфное решение $\psi(\tau)$ без полюсов на границе кольи, Тогда

$$
\chi_{r_{2}}(\psi)=\chi_{r_{1}}(\psi)-2 \#\left(\text { полюсы функиии }\left.\psi\right|_{r_{1}<|\tau|<r_{2}}\right) .
$$

Доказательство. Ненулевое решение $v(\tau)$ уравнения в вариациях вдоль $\psi$ есть функция со значениями в касательном расслоении к сфере Римана, голоморфная в окрестности кольца $A_{r_{1} r_{2}}$ и не обращающаяся в нуль. Пусть $p$ - комплексная координата на $\mathbb{C}$. Она задает стандартную тривиализацию касательного расслоения $T \overline{\mathbb{C}}$ над $\mathbb{C}$. Функция $v(\tau)$ со значениями в $T \overline{\mathbb{C}}$, записанная в стандартной тривиализации, мероморфна, и мы будем ее обозначать тем же символом $v(\tau)$. Покажем, что функция $v(\tau)$ имеет те же полюсы и той же кратности, что и функция $\psi^{2}(\tau)$. Это вместе с принципом аргумента докажет предложение 3.11. Пусть $\tau^{\prime} \in \operatorname{Int}\left(A_{r_{1} r_{2}}\right)-$ полюс функции $\psi$. Пусть $V \subset \overline{\mathbb{C}} \backslash 0$ - произвольная малая окрестность бесконечности, точнее, дополнение к большому диску с центром в нуле, а $D \subset A_{r_{1} r_{2}}$ - малый замкнутый диск с центром в $\tau^{\prime}$, такой, что $\psi(D) \subset V$. Рассмотрим вспомогательную тривиализацию касательного расслоения $T \overline{\mathbb{C}}$ над $V$, заданную с помощью карты $\widetilde{p}=1 / p$. Напомним, что $T \overline{\mathbb{C}}$-значное решение уравнения в вариациях голоморфно на замкнутом диске $D$ и в новой тривиализации записывается как $\widetilde{v}(\tau)=\psi^{-2}(\tau) v(\tau)$. По построению функция $\widetilde{v}(\tau)$ голоморфна и не обращается в нуль на диске $D$ и $v(\tau)=\widetilde{v}(\tau) \psi^{2}(\tau)$. Следовательно, функция $v(\tau)$ имеет те же полюсы и той же кратности, что и функция $\psi^{2}(\tau)$. Предложение 3.11 доказано.

Справедливость леммы 3.8 вытекает из предложений $3.9,3.10$ и 3.11 .

Лемма 3.12. Пусть функиии $\left.\left.\psi_{1}\right|_{S_{\tau}^{1}} u \psi_{2}\right|_{S_{\tau}^{1}}$ либо принимают значения, лежащие по разные стороны от единичной окружности $S_{p}^{1}$, либо $\psi_{j}\left(S_{\tau}^{1}\right) \subset S_{p}^{1}$ при некотором $j=1,2$. Тогда число вращения рассматриваемого уравнения класса Д равно абсииссе перемычки: $\rho=a$.

Ниже мы выведем лемму 3.12 из леммы 3.8 и следующего предложения.

Предложение 3.13. В условиях леммы 3.12

$$
\left.\left|\psi_{1}\right|\right|_{\overline{D_{1}}} \leqslant 1,\left.\quad\left|\psi_{2}\right|\right|_{\overline{D_{1}}} \geqslant 1 .
$$

Доказательство. Рассмотрим функцию $\phi=\psi_{1} / \psi_{2}$. Она мероморфна на $\mathbb{C}$ и обладает следующими свойствами:

$$
\phi \neq 1, \quad \phi(0)=0 ; \quad \text { либо }\left.|\phi|\right|_{S_{\tau}^{1}} \leqslant 1, \quad \text { либо }\left.|\phi|\right|_{S_{\tau}^{1}}>1 .
$$

Утверждение (3.7) следует из условий леммы 3.12 и замечания 3.7. Мы разберем сначала первый случай, когда $\left.|\phi|\right|_{S_{\tau}^{1}} \leqslant 1$, а затем покажем, что второй случай, 
когда $\left.|\phi|\right|_{S_{\tau}^{1}}>1$, невозможен. Рассмотрим разность $\phi(\tau)-1$. Она мероморфна и не обращается в нуль на $\mathbb{C}$, а приращение ее аргумента вдоль окружности $S_{\tau}^{1}$ равно нулю, так как $\phi\left(S_{\tau}^{1}\right) \subset \overline{D_{1}}$. Следовательно, функция $\phi-1$ не имеет полюсов в единичном диске. Значит, $\left.|\phi|\right|_{D_{1}} \leqslant 1$ по принципу максимума и так как последнее неравенство выполнено на границе диска по предположению. Итак, $\left|\psi_{1}\right| \leqslant\left|\psi_{2}\right|$ на единичном диске. С другой стороны, на единичной окружности выполнено одно из неравенств $\left|\psi_{1}\right| \leqslant 1 \leqslant\left|\psi_{2}\right|,\left|\psi_{2}\right| \leqslant 1 \leqslant\left|\psi_{1}\right|$ в силу условия леммы 3.12. Следовательно, первое неравенство выполнено на всем замкнутом единичном диске, и предложение 3.13 доказано. Пусть теперь имеет место второй случай: $\left.|\phi|\right|_{S_{\tau}^{1}}>1$, т. е. $\left.\left|\phi^{-1}\right|\right|_{S_{\tau}^{1}}<1$. Разность $\phi^{-1}(\tau)-1$ мероморфна и не обращается в нуль на $\mathbb{C}$, а приращение ее аргумента вдоль окружности $S_{\tau}^{1}$ равно нулю, как и выше. В то же время рассматриваемая разность имеет полюс в нуле, что противоречит принципу аргумента. Полученное противоречие доказывает невозможность рассматриваемого случая и завершает доказательство предложения 3.13 .

Доказательство леммы 3.12. Имеем $\left|\psi_{1}\right|_{D_{1}} \leqslant 1$ в силу предложения 3.13. Отсюда и из леммы 3.8 следует утверждение леммы 3.12 .

Лемма 3.14. Пусть на единичной окружности $S_{\tau}^{1}$ выполнены неравенства $\left|\psi_{1}\right|,\left|\psi_{2}\right| \leqslant 1$. Тогда $\rho \leqslant a<0$, где a- -абсиисса рассматриваемой перемьчки.

Доказательство. Неравенство $\rho \leqslant a$ следует из леммы 3.8. Достаточно показать, что $\rho<0$; тогда $a<0$ в силу следствия 3.6.

Предложение 3.15. В условиях леммы 3.14 число вращения $\rho$ равно приращению аргумента разности $\psi_{2}-\psi_{1}$ вдоль единичной окружности.

Доказательство. Напомним, что $\psi_{1}(\tau) \neq \psi_{2}(\tau)$ при всех $\tau \in \mathbb{C}$. Рассмотрим отображения потока $H_{1, \tau}: \overline{\mathbb{C}} \times\{1\} \rightarrow \overline{\mathbb{C}} \times\{\tau\}$ уравнения Риккати $(2.7)$ при $\tau \in S_{\tau}^{1}$. Преобразования $H_{1, \tau}$ являются конформными автоморфизмами единичного диска, и $H_{1, \tau}\left(\psi_{j}(1), 1\right)=\left(\psi_{j}(\tau), \tau\right)$ при $j=1,2$, так как $\psi_{j}$ суть решения уравнения (2.7). Отсюда следует, что геодезическая метрики Пуанкаре на $D_{1}$, соединяющая точки $\psi_{1}(1)$ и $\psi_{2}(1)$, переходит в геодезическую $\gamma_{\tau}$, соединяющую точки $\psi_{1}(\tau)$ и $\psi_{2}(\tau)$. Каждый из концов геодезической $\gamma_{\tau}$ пробегает единичную окружность, делая $\rho$ полных оборотов, когда $\tau$ пробегает единичную окружность $S_{\tau}^{1}$ в положительном направлении. Это следует из определения числа вращения. С другой стороны, приращение аргумента направляющего единичного вектора геодезической $\gamma(\tau)$ в точке $\psi_{1}(\tau)$ равно приращению аргумента прямолинейного вектора на евклидовой плоскости $\mathbb{R}^{2}=\mathbb{C} \subset \overline{\mathbb{C}}$, направленного из точки $\psi_{1}(\tau)$ в точку $\psi_{2}(\tau)$. Это следует из того, что угол между рассматриваемыми векторами меньше $\pi$ при всех $\tau$. Итак, приращение аргумента функции $\psi_{2}(\tau)-\psi_{1}(\tau)$ равно числу вращения. Предложение 3.15 доказано.

Предложение 3.16. В условиях леммъ 3.14 приращение аргумента разности $\psi_{2}-\psi_{1}$ вдоль единичной окруюнности отрицательно.

Доказательство. Рассмотрим матричнозначную функцию $H(\tau)$ нормализующей замены, приводящей линейное уравнение (2.8) к нормальной форме (3.1): $z=H(\tau) w$. Функция $H(\tau)$ голоморфна на $\mathbb{C}$ и принимает значения в обратимых матрицах. Напомним, что $\psi_{j}=h_{2 j} / h_{1 j}$ при $j=1,2$ в силу предложения 
2.10. Таким образом,

$$
\psi_{2}-\psi_{1}=\frac{h_{11} h_{22}-h_{21} h_{12}}{h_{11} h_{12}} .
$$

Числитель в правой части последнего равенства есть определитель обратимой матричнозначной функции $H$, а значит, он не обращается в нуль на $\mathbb{C}$. Значит, приращение аргумента рассматриваемой разности равно минус сумме количеств нулей (с учетом кратностей) функций $h_{11}$ и $h_{12}$ в единичном диске. Последняя сумма положительна, так как $h_{12}(0)=0$. Следовательно, рассматриваемое приращение аргумента отрицательно. Предложение 3.16 доказано.

Окончание доказательства леммы 3.14. Неравенство $\rho<0$ следует из предложений 3.15 и 3.16. Это вместе с рассуждениями из начала доказательства леммы 3.14 доказывает эту лемму.

Окончание доказательства теоремы 1.2. Мы уже доказали, что абсциссы перемычек целочисленны. Напомним, что для каждой перемычки каждая из соответствующих функций $\left.\psi_{j}\right|_{S_{\tau}^{1}}, j=1,2$, принимает значения либо по одну сторону от единичной окружности, либо на самой окружности. Имеет место один из следующих трех случаев:

(i) функции $\left.\psi_{j}\right|_{S_{\tau}^{1}}, j=1,2$, принимают значения, лежащие по разные стороны от единичной окружности; сюда мы включаем и случай, когда $\left|\psi_{j}\right|_{S_{\tau}^{1}} \equiv 1$ при некотором $j=1,2$;

(ii) $\left|\psi_{j}\right|_{S_{\tau}^{1}}<1$ при всех $j=1,2$;

(iii) $\left|\psi_{j}\right|_{S_{\tau}^{1}}>1$ при всех $j=1,2$.

В первом случае $\rho=a$ по лемме 3.12. Во втором случае $\rho \leqslant a<0$ по лемме 3.14. Третий случай сводится к второму заменой переменных $(x, t) \mapsto(-x, t+\pi)$ $\left((p, \tau) \mapsto\left(p^{-1},-\tau\right)\right.$ в координатах $\left.(p, \tau)\right)$. Последняя замена переводит уравнение класса Д в то же уравнение, но меняет знак параметра $a$ и числа вращения. Отсюда и из предыдущего утверждения следует, что в третьем случае имеет место неравенство $0<a \leqslant \rho$. При $a=0$ имеем $\rho=0$ в силу предложения 3.5. Это доказывает теорему 1.2 .

3.4. Экспериментальный факт А в общем случае: текущее состояние дел. Экспериментальный факт А, утверждающий, что $\rho=a$ для каждой перемычки, доказан при $|\nu| \leqslant 1$ (теорема 1.2). Ожидается, что он верен при любом $\nu \neq 0$.

Теорема 3.17. Для каждой перемычки разность $\rho-a$ всегда четна. Если она не равна нулю, то либо $\rho<a<0$, либо $0<a<\rho$.

Теорема 3.17 следует из леммы 3.8 и теоремы 1.2 .

Пусть $(\nu, a, s)$ - перемычка. Пусть $\psi_{1}, \psi_{2}$ - голоморфные в нуле решения соответствующего уравнения Риккати (2.7) из предложения 2.10.

Как показано ниже, равенство $\rho=a$ имеет место при следующем условии:

Условие (*) на уравнение Риккати (2.7). Либо $\psi_{1}(\tau)$ не имеет полюсов в единичном диске и $\left.\left|\psi_{1}\right|\right|_{S_{\tau}^{1}} \leqslant 1$, либо $\psi_{2}(\tau)$ не имеет нулей в единичном диске и $\left.\left|\psi_{2}\right|\right|_{S_{\tau}^{1}} \geqslant 1$.

Лемма 3.18. Пусть $(\nu, a, s)-$ перемъчка $(s \neq 0)$. Тогда $\rho=a$, если и только если соответствующее уравнение Риккати удовлетворяет условию (*). 
Лемма 3.18 следует из леммы 3.8, аналог которой справедлив и в случае, когда $\left.\left|\psi_{2}\right|\right|_{S_{\tau}^{1}} \geqslant 1$, с заменой функции $\psi_{1}$ на $\psi_{2}$, а количества полюсов - на количество нулей.

Гипотеза С. Условие $(*)$ выполнено для всякой перемычки.

Экспериментальный факт А эквивалентен гипотезе С в силу леммы 3.18.

Авторы благодарны В. М. Бухштаберу, Э. Жису, Ю. С. Ильяшенко, А. В. Клименко и О. Л. Ромаскевич за полезные обсуждения.

\section{ЛиТЕРАТУРА}

[1] В. И. Арнольд, Геометрические методь в теории обыкновенных дифберенциальных уравнений, РХД, МЦНМО, ВКМ НМУ, М., 1999.

[2] В. И. Арнольд, Ю. С. Ильяшенко, Объкновенные дифференииальные уравнения, в кн.: Динамические системы-1, Итоги науки и техники, Современные проблемы математики. Фундам. направления, т. 1, ВИНИТИ, М., 1985, 7-140.

[3] В. М. Бухштабер, О. В. Карпов, С. И. Тертычный, Система на торе, моделирующая динамику перехода Джсзефсона, УМН, 67:1(403) (2012), 181-182.

[4] В. М. Бухштабер, О. В. Карпов, С. И. Тертычный, Эффект квантования числа вращения, ТМФ, 162:2 (2010), 254-265.

[5] В. М. Бухштабер, О. В. Карпов, С. И. Тертычный, Особенности динамики джозебсоновского перехода, смещенного синусоидальным СВЧ-током, Радиоэлектроника и электротехника, 51:6 (2006), 757-762.

[6] В. М. Бухштабер, О. В. Карпов, С. И. Тертычный, О свойства дифференииального уравнения, описывающего динамику сильношунтированного перехода Дюозефсона, УМH, 59:2 (2004), 187-188.

[7] Ю. С. Ильяшенко, Лекиии летней школь «Динамические системь», Попрад (Словакия), 2009.

[8] Ю. С. Ильяшенко, Д. А. Рыжов, Д. А. Филимонов, Захват фазы для уравнений, описывающих резистивную модель джозефсоновского перехода, и их возмущений, Функц. анализ и его прил., 45:3 (2011), 41-54.

[9] Ю. С. Ильяшенко, А. Г. Хованский, Группъ Галуа, операторъ Стокса и теорема Рамиса, Функц. анализ и его прил., 24:4 (1990), 31-42.

[10] К. К. Лихарев, Б. Т. Ульрих, Системы с джсозесоновскими контактами: основы теории, Изд-во МГУ, М., 1978.

[11] Н. Н. Лузин, О методе приближенного интегрирования акад. С. А. Чапльгина, УMH, 6:6(46) (1951), 3-27.

[12] W. Balser, W. B. Jurkat, D. A. Lutz, Birkhoff invariants and Stokes' multipliers for meromorphic linear differential equations, J. Math. Anal. Appl., 71:1 (1979), 48-94.

[13] A. Barone, G. Paterno, Physics and Applications of the Josephson Effect, John Wiley and Sons, New York-Chichester-Brisbane-Toronto-Singapore, 1982.

[14] R. L. Foote, Geometry of the Prytz Planimeter, Rep. Math. Phys., 42:1-2 (1998), 249-271.

[15] J. Guckenheimer, Yu. S. Ilyashenko, The duck and the devil: canards on the staircase, Moscow Math. J., 1:1 (2001), 27-47.

[16] S. Shapiro, A. Janus, S. Holly, Effect of microwaves on Josephson currents in superconducting tunneling, Rev. Mod. Phys., 36 (1964), 223-225.

[17] W. B. Jurkat, D. A. Lutz, A. Peyerimhoff, Birkhoff invariants and effective calculations for meromorphic linear differential equations, J. Math. Anal. Appl., 53:2 (1976), 438470. 
[18] A. Klimenko, O. Romaskevich, Asymptotic properties of Arnold tongues and Josephson effect, Moscow Math. J., 14:2 (2014), 367-384.

[19] Y. Sibuya, Stokes phenomena, Bull. Amer. Math. Soc., 83:5 (1977), 1075-1077.

CNRS, France (UMR 5669 (UMPA, ENS de Lyon)

Поступило в редакцию and UMI 2615 (Laboratoire J.-V. Poncelet)) 30 января 2013 г.

Независимый московский университет

Национальный исследовательский университет «Высшая школа экономики» e-mail: aglutsyu@ens-lyon.fr

CNRS, Institut de Recherche Mathématique de Rennes (UMR 6625), France e-mail: victor.kleptsyn@univ-rennes1.fr Национальный исследовательский университет «Высшая школа экономики» Московский физико-технический институт e-mail:mityafil@gmail.com

Национальный исследовательский университет «Высшая школа экономики» e-mail: ilya@schurov.com 